\title{
Thin film metal sensors in fusion bonded glass chips for high-pressure microfluidics
}

\author{
Martin Andersson ${ }^{1}$, Johan $\mathrm{Ek}^{2}$, Ludvig Hedman, ${ }^{2}$ Fredrik Johansson ${ }^{2}$, Viktor Sehlstedt ${ }^{2}$, Jesper \\ Stocklassa $^{2}$, Pär Snögren ${ }^{2}$, Victor Pettersson ${ }^{2}$, Jonas Larsson ${ }^{2}$, Olivier Vizuete ${ }^{2}$, Klas Hjort ${ }^{1}$ and \\ Lena Klintberg \\ Department of Engineering Sciences, Uppsala University, Box 534, 75121 Uppsala, Sweden \\ ${ }^{1}$ Corresponding author: martin.andersson@angstrom.uu.se; klas.hjort@angstrom.uu.se \\ ${ }^{2}$ Project member in the Advanced Level Undergraduate Project course on Micro- and \\ Nanotechnology, Uppsala University
}

\begin{abstract}
High-pressure microfluidics offers fast analyses of thermodynamic parameters for compressed process solvents. However, microfluidic platforms handling highly compressible supercritical $\mathrm{CO}_{2}$ are difficult to control, and on-chip sensing would offer added control of the devices. Therefore, there is a need to integrate sensors into highly pressure tolerant glass chips. In this paper, thin film Pt sensors were embedded in shallow etched trenches in a glass wafer that was bonded with another glass wafer having microfluidic channels. The devices having sensors integrated into the flow channels sustained pressures up to 220 bar, typical for the operation of supercritical $\mathrm{CO}_{2}$. No leakage from the devices could be found. Integrated temperature sensors were capable of measuring local decompression cooling effects and integrated calorimetric sensors measured flow velocities over the range $0.5-13.8 \mathrm{~mm} / \mathrm{s}$. By this, a better control of highpressure microfluidic platforms has been achieved.
\end{abstract}




\section{Introduction}

Having a viscosity comparable to gases and a liquid-like density, supercritical $\mathrm{CO}_{2}$ is a benign, non-toxic and compressible solvent for several types of organic compounds. It is used for a multitude of applications, e.g., high-value products in the food industry [1], purification and analytics in the pharmaceutical industry [2] and, particle formation and loading of pharmaceutical compounds to carrier substrates [3-5].

Use of the fluid requires knowledge of multicomponent thermodynamic parameters like solubility. Conventional methods [6] used to determine high-pressure phase equilibriums are generally labour intensive, time-consuming and demand special safety precaution. Therefore, high-pressure microfluidics has emerged as a potentially fast and efficient alternative for the screening of such parameters [7]. But to increase its applicability, better control is needed of the highly variable fluids inside the chips. In this paper, the integration of sensor elements into highpressure microfluidic channels is explored for applications with supercritical $\mathrm{CO}_{2}$.

Thin film sensors and electrodes in microfluidic chips offer a large degree of added functionality. For low-pressure applications, this has been exemplified by systems for detection and control, e.g., systems that are impedance based [8] or electrochemical [9, 10, 12], and as microplasma sources [11]. With electrodes in the chips having direct access to the fluids, systems can have both a small footprint and a low dead volume.

Several research groups have demonstrated glass chips with integrated metal thin films [10-11, 13-15]. One fabrication method that we have followed involves etching a trench for the metal layer followed by a heat treatment at $625^{\circ} \mathrm{C}$ [14]. However, such systems have only been demonstrated at low pressures. As one part of this paper, we investigate the conditions necessary to fulfill for chips with embedded Pt thin films to withstand the pressures needed for supercritical $\mathrm{CO}_{2}$.

A supercritical fluid shows large variations in a number of physical qualities such as density, viscosity, thermal conductivity, and heat capacity, around its critical point, which for $\mathrm{CO}_{2}$ is at $31.1^{\circ} \mathrm{C}$ and 73.9 bar (7.39 MPa) [16]. The density must be controlled, as it is a key parameter affecting other properties, like the solubility of compounds. Further, $\mathrm{CO}_{2}$ is a compressible fluid that can, during decompression, be subject to cooling by both the Joule-Thompson effect and by liquid-vapor phase transformations. For microfluidic platforms having flow restrictors and complex designs; $\mathrm{CO}_{2}$ will be in a non-equilibrated state. Also, at near-critical conditions, a heterogeneous gas-liquid flow may add to the complexity [17-18]. To control such a fluid systems, it is necessary to have precise control of the temperature. The large surface to volume ratio in microchips allows for good heat transfer and common approaches for external temperature control includes having chips mounted on heated Cu blocks [19], immersed in oil [20] or mounted on resistive heat stages [21]. For all such systems, the temperature is measured externally and globally. Consequently, by adding local sensing capability, much-needed insight regarding the actual state of the fluid in the chip can be given.

This paper presents two high-pressure chips. To expose the cooling effects connected to decompression, a pressure drop chip with temperature sensors is used. The other chip is a calorimetric flow sensor. This sensor principle has been widely explored in the field of MEMS and works by measuring the temperature increase of a fluid as it passes over a heater [22-25]. While the response of such sensor principle is primarily dependent on flow velocity, other factors, including the fluid density and heat capacity, also affect the response [26]. By these two chips, it is explored how internal integrated temperature and flow velocity sensing can be achieved in 
transparent microfluidic chips at the high pressures required for supercritical $\mathrm{CO}_{2}$.

\section{Background}

\section{Metal layers in bond interfaces}

Models have been developed to describe how wafer bonding is influenced by surface variations [27]. With protrusions, gaps will form, figure 1. By such a model, wafers will bond, and conform to the gap, if the elastic strain energy required is less than the lowering in surface energy as the wafers adhere [28]. With small enough particles, elastomechanical instability allows for embedment, resulting in a gap of length $2 \mathrm{R}$, around the particle that will be in the same order of length as the diameter, $2 \mathrm{~h}$, of the particle, figure $1 \mathrm{a}$. However, as soon as the particle is larger than a critical size, the gap size will be in the order of the thickness of the wafer. Specifically, when embedded in-between two $1.1 \mathrm{~mm}$ thick borosilicate glass wafers, with Young's modulus of 64 GPa, Poisson's ratio of 0.2 [29], and a surface energy in the range of 80 to $160 \mathrm{~mJ} / \mathrm{m}^{2}$, a rigid particle has a critical height in the range of 0.18 to $0.26 \mu \mathrm{m}$ (following Eq. 6 in [27]). However, as soon as the particle is larger than its critical value, the gap radius will grow to $3 \mathrm{~mm}$, or more (combining Eqs. 5 and 6 in [27]).

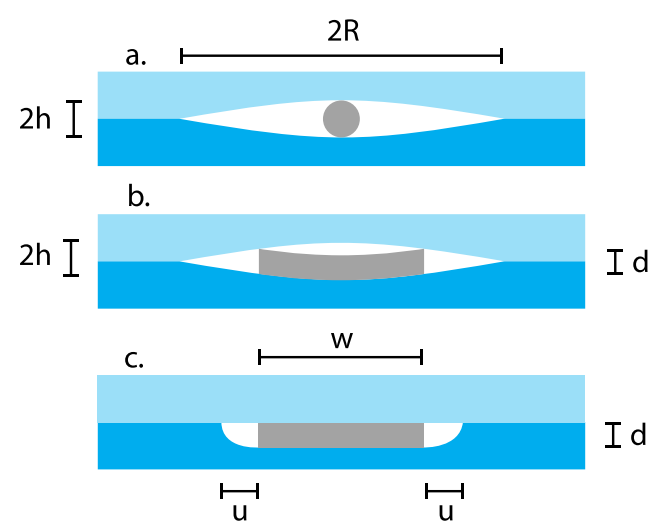

Figure 1 Schematic cross-sections: In a, a particle in the bond interface produces a gap. In b, a flat metal layer deposited on top of a wafer in the bond interface produces a similar gap. In c, the metal layer is instead deposited in an etched trench.

For a flat metal layer on one of the wafers, the critical height of the film will be lower, figure $1 \mathrm{~b}$. Due to the sinusoidal-shaped gap, the metal layer thickness, $\mathrm{d}$, needs to be less than $2 \mathrm{~h}$ in order to give the same gap size. The metal layer width, $w$, will determine the contact points, and hence the $\mathrm{d} / \mathrm{h}$ ratio. By forming an etched trench and lowering the metal layers into the substrate, the problem can be evaded, figure $1 \mathrm{c}$. This has been a successful approach for low-pressure applications [10-11, 13-14]. Gaps, $\mathrm{u}$, formed along the edges by using such methods are then dependent on the dimensional mismatch between the trench and the metal, giving rise to possible voids around the metal. If these voids can be kept small, a high-pressure endurance of the chips should be possible; also when having many interconnects on a single chip.

The pressure tolerance of chips is dependent on several factors, including the geometry and wafer bonding scheme [30, 31]. For semi-circular channels, the largest stresses are generally seen along the bond interface. By modeling pressurized channels using finite element analysis, the stress along the channel can be calculated from the fracture pressure, and a comparable, geometry 
independent measurement of strength can be made.

Flow of $\mathrm{CO}_{2}$ in microfluidics

$\mathrm{CO}_{2}$ is highly compressible in the region around the critical point and the fluid flow to and from the microfluidic chip depend on several variables. Given an equilibrated system as the experimental setup sketched in figure 2, the pressure difference, caused by a change in volume between two pistons, is driving the flow.

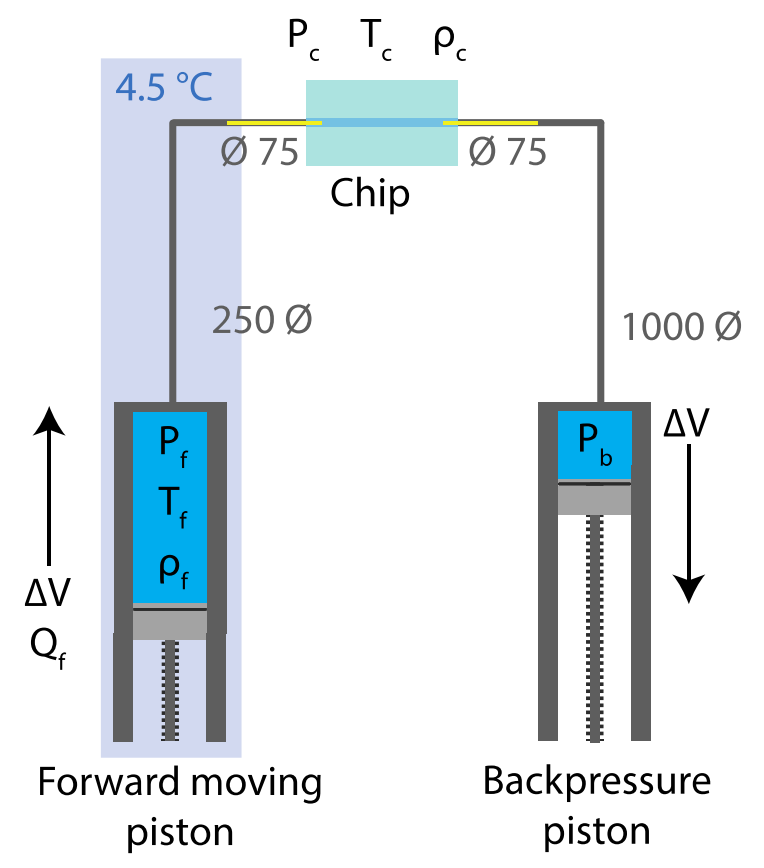

Figure 2 Experimental setup for chip characterization showing the forward moving piston, the chip, and the backpressure piston. System parameters at these three positions are shown. Two capillaries (orange) of $10 \mathrm{~cm}$ connect the chip to stainless steel tubing (gray) having the inner diameter given in $\mu \mathrm{m}$. Cooling (light blue) is applied to the forward piston and the tubing before the chip.

Keeping the pressure difference between the two pistons small allows the system to be close to equilibrium. A decrease in volume of the forward moving piston corresponds to a volumetric flow rate of $\mathrm{Q}_{\mathrm{f}}$. However, this is not the same volumetric flow rate as in the chip. Hence, it is convenient to describe the piston flow in terms of mass flow, $\dot{\mathrm{m}}$. While maintaining pressure, $\mathrm{P}_{\mathrm{f}}$, and temperature, $\mathrm{T}_{\mathrm{f}}$, in the piston, $\dot{\mathrm{m}}$ can be calculated as

$\dot{\mathrm{m}}=\mathrm{Q}_{\mathrm{f}} \rho_{\mathrm{f}}$

with the density, $\rho_{\mathrm{f}}$, derived from $\mathrm{P}_{\mathrm{f}}, \mathrm{T}_{\mathrm{f}}$, and an equation of state [16] valid under these conditions. The second piston can be set to regulate a backpressure, $P_{b}$. The difference between $P_{f}$ and $\mathrm{P}_{\mathrm{b}}$ comes from the resulting pressure drop between the two pistons, $\Delta \mathrm{P}_{\mathrm{fb}}$, while maintaining a flow between the pistons. Excluding the chip, the capillaries are the main source of flow restriction and $\Delta \mathrm{P}_{\mathrm{fb}}$ can be estimated by considering the density $\rho_{\mathrm{c}}$ at the pressure, $\mathrm{P}_{\mathrm{c}}$, and temperature, $\mathrm{T}_{c}$, found in and around the chip, and then applying the Hagen Poiseuille's equation, 
$\Delta \mathrm{P}_{\mathrm{fb}}=\frac{128 \mu \mathrm{Lm}}{\pi \mathrm{D}_{\mathrm{H}}^{4} \rho_{\mathrm{c}}}$

where $L$ is channel length, $\mu$ is the viscosity [32] and $D_{H}$ is the hydraulic diameter, calculated from the perimeter and cross section of the channel. Though strictly valid for incompressible flow, it has been used with a good experimental agreement in other works [7]. For chips without pronounced flow restrictors, $\mathrm{P}_{\mathrm{f}}, \mathrm{P}_{\mathrm{b}}$, and $\mathrm{P}_{\mathrm{c}}$ can be regarded as approximately equal.

The corresponding flow velocity in the chips, $\mathrm{v}$, it is given by

$\mathrm{v}=\frac{\dot{\mathrm{m}}}{\mathrm{A} \rho_{\mathrm{c}}}$

where A is the channel cross section in the chip.

\section{Materials and method}

\section{Design}

Embedded electrodes are deposited on a wafer and then sealed by bonding another wafer with microfluidic channels on top. As such procedure must be compatible with the bonding method used to create strong high-pressure resistant chips, it limits the material choices. Pt shows a high degree of corrosion resistance and is compatible with both $\mathrm{HNO}_{3}$ and $\mathrm{KOH}$ [33] at the temperatures, $65-85^{\circ} \mathrm{C}$, used in the bonding scheme. Pt is also resistant to the thermal treatments needed after bonding. By depositing $100 \mathrm{~nm}$ thick and $10 \mu \mathrm{m}$ wide Pt layers, it is possible to make resistive elements in the $\mathrm{k} \Omega$ range, having a footprint of 110 by $100 \mu \mathrm{m}$, thus fitting well within a fluid channel. The interconnects to the sensing elements, connected in a 4-point configuration, and heater, should be wide in order to give a low resistance, but at the same time narrow not to lower the high-pressure strength of the chips since the bond strength between metal and the bonded glass wafer probably will be the weakest link.

Two different kinds of demonstrator chips are made in this paper; a pressure drop chip and a flow sensor chip, and schematic drawings of these chips are found in figure 3. The outer dimensions of both chip designs were 15 by $5 \mathrm{~mm}$. Both of the designs have semi-circular channels of $160 \mu \mathrm{m}$ depth and $360 \mu \mathrm{m}$ width, and are open to the sides of the chip, thereby forming inlets that give access to outer diameter $150 \mu \mathrm{m}$ silica capillaries, forming a fluid interface. 

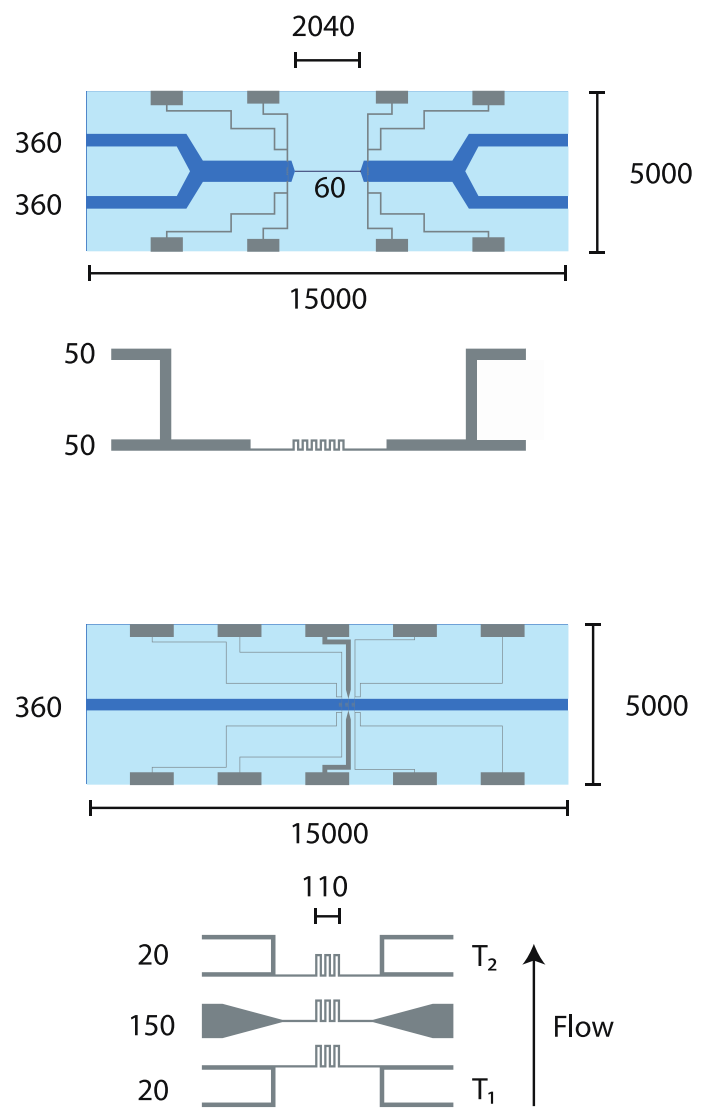

Figure 3 Drawings of the pressure drop chip (top) and flow sensor chip (bottom). Widths of chips, channels and conductors are given in $\mu \mathrm{m}$. The sensing elements and the interconnects over the channel interface are $10 \mu \mathrm{m}$ wide. For the flow sensing elements, the temperature sensor closest to the flow is denoted $\mathrm{T}_{1}$ and the one further away is denoted $\mathrm{T}_{2}$.

The ratio between channels and bonded areas is 0.93 and 0.86 for the flow sensor and pressure restrictor, respectively. On the pressure drop chip, a small $5.4 \mu \mathrm{m}$ deep and $60 \mu \mathrm{m}$ wide restrictor channel is in-between the wide fluid inlet and outlet channels. At the ends of the restrictor, the channel width is larger, $660 \mu \mathrm{m}$. By this design, a significant pressure drop is formed in the chip when flowing $\mathrm{CO}_{2}$, much more than the $\Delta \mathrm{P}_{\mathrm{fb}}$ of 0.24 bar of the surrounding system. Using Eq. (2) and $\mathrm{D}_{\mathrm{H}}$ calculated from the perimeter and cross section of the channel, $\Delta \mathrm{P}_{\mathrm{fb}}$ increases up to 11 bar. The pressure drop will cause $\mathrm{CO}_{2}$ to expand, resulting in cooling. To investigate this behavior, thin film temperature elements are positioned right next to both the inlet and the outlet of the restrictor.

The other design, the flow sensor chip, consists of a straight channel of the same dimensions as the inlets and holds a flow sensor in its center. The sensor consists of a heating element and two temperature elements on either side of the heater in the flow direction. All temperature elements, in both designs, have 4 conductors leading to each sensor element, and by a 4-point measurement scheme, this allows for the localized measurement of resistance in the channel.

\section{Fabrication}

The devices were manufactured by structuring two glass wafers, having metal conductors on one side and etched channels on the other. The process scheme includes wet etching using HF, fusion 
bonding and a thermal treatment at $625^{\circ} \mathrm{C}$ for $6 \mathrm{~h}$. In this section, the focus is on the fabrication of the electrode wafers and the bonding scheme.

Borosilicate glass (Borofloat 33, Schott) $1.1 \mathrm{~mm}$ thick and 4" diameter, were first cleaned in a Megasonic cleaning bath (PCT Systems Inc.) for $1.5 \mathrm{~min}$ with a solution of $25 \mathrm{wt} . \% \mathrm{KOH}$ at $65^{\circ} \mathrm{C}$. This was followed by immersion in $69 \mathrm{wt} . \% \mathrm{HNO}_{3}$ at $80{ }^{\circ} \mathrm{C}$ for $10 \mathrm{~min}$. The electrodes, deposited on one of the wafers, were made from Pt using a lift-off process. First, a 1 min $1000 \mathrm{~W}$ $\mathrm{O}_{2}$ plasma cleaning process was done (Plasma Processor 300, Tepla) which was followed by the addition of an adhesion promoting primer, HMDS, in a priming oven (Star 2000, Star). $1 \mu \mathrm{m}$ resist (1813, Shipley) was then added by spinning and baking at $115^{\circ} \mathrm{C}$ for $1 \mathrm{~min}$ on a hotplate. The resist was exposed and then treated in an image reversal processing oven (58TA, Yield Engineering Systems Inc) and flood exposed. To form trenches, the wafers were etched in buffered oxide etch (BOE 1:7, J.T.Baker) to an aimed depth of $100 \mathrm{~nm}$. Following rinsing and spin-drying, the depth of the trench was checked using profilometry (DektakXT, Bruker). The metal film was deposited using sputtering (K675XD, Emitech) with first a $10 \mathrm{~nm}$ thick Ti adhesion layer followed by a $100 \mathrm{~nm}$ thick Pt layer. After deposition, the resist was lifted off in an ultrasonic bath with acetone for $10 \mathrm{~min}$. This was followed by rinsing in IPA and $\mathrm{O}_{2}$ plasma cleaning for $10 \mathrm{~min}$ at $1000 \mathrm{~W}$.

The other wafer, containing the fluid channels, was wet etched with HF using a mask made from HDMS primer, spray resist (AZ9260, Micro Chemicals), and a $1 \mu \mathrm{m}$ thick sputtered molybdenum masking layer. For the deep and shallow channels, 49 wt.\% and 12.3 wt.\% HF was used, respectively. The full fabrication method of the fluid channels follows [30].

The bonding was performed in ambient air at atmospheric pressure, and alignment of the wafers was done manually over a stereoscope (SMZ800, Nikon) by first adding three $0.5 \mathrm{~cm}$ wide tape insertions (SWT 10, Nitto) between the wafer edges and then pressing firmly on the center, thereby starting the bonding process. As this locks the wafers in place, the tape was removed and all of the wafer area bonds. Bonded wafers were placed on SiN covered dummy wafers in a vertical oven (Micro TF-6, Koyo Lindberg) and were heat-treated at $625^{\circ} \mathrm{C}$ for $6 \mathrm{~h}$ with a ramp up and ramp down at $1{ }^{\circ} \mathrm{C} / \mathrm{min}$. No weights were used on the wafers. After heat treatment, the wafers were diced using a dicing saw (DAD 361, Disco). By such action, both fluid inlets and metal interconnects are exposed on the sides of the chips. To better expose the electrode edges before making the electrical connections, the electrodes were lightly polished with SiC paper (600 grit).

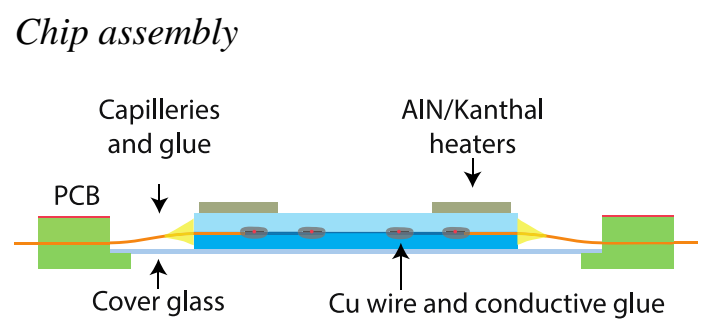

Figure 4 Cross-section of a chip mounted on a cover glass and a PCB fixture. AlN/Kanthal heaters were mounted on top of the chip.

$75 \mu \mathrm{m}$ inner diameter and $150 \mu \mathrm{m}$ outer diameter capillaries were manually inserted 2-2.5 mm into the inlets on the sides of the chips and glued using 2-component epoxy glue (Araldite Rapid, Huntsman Advanced Materials). To allow for transmission lightning, the chips were then placed 
on $100 \mu \mathrm{m}$ thick cover glass slabs. When the external temperature of chips needed to be raised, resistive Kanthal wirings were used. To achieve good thermal conductivity, the wirings were wound around AlN substrates and connected to the chips with thermal grease. As the chips required both electrical connections and a high-pressure fluid interface, a mounting fixture was made. It consisted of two printed circuit boards, PCBs, with centered holes. The cover glass with the chip was mounted in between the PCBs, figure 4. To gain electrical access to the chips, 200 $\mu \mathrm{m}$ thick $\mathrm{Cu}$ wires were on one end soldered onto pads on the top PCB, and on the other end glued to the sides of the chip using electrically conductive 2-component epoxy glue (CW2400, CircuitWorks). A flat cable connected to the top PCB provided electrical access to the instrumentation.

\section{Experimental Setup}

To characterize the chips, a high-pressure test system was used that consisted of two highpressure piston pumps (100 DM, ISCO Teledyne), cooling baths and a high-speed camera mounted on a microscope. The forward moving piston, figure 2, contained $\mathrm{CO}_{2}(99.9995 \%$, AGA). The tubing and forward moving piston were cooled by a compressor (RK-20, Lauda) connected to a $20 \mathrm{~L}$ cooling bath held at $4.5^{\circ} \mathrm{C}$. The large thermal buffer was used for increased stability. The chips, mounted on a fixture with electrical and fluid interfaces, where held about $5 \mathrm{~cm}$ above this cooling bath and a high brightness LED was mounted just above the water line. To measure the temperature of the forward moving piston, $T_{f}$, a Pt100 sensor was used in 4-point configuration together with a data acquisition control unit (34970A, Agilent). The high-speed camera (Miro 320, Phantom Vision) was mounted on a stereoscope (SMZ800, Nikon) above the chip fixture, forming a high-speed transmission light setup.

The electric resistance of the integrated temperature elements was measured using another data acquisition control unit (9219, National Instruments) in 4-point configuration and the data was recorded using LabView. The heating element of the flow sensor was operated using a current controlled power supply (QL335TP, TTi).

As the chips needed to be observed with the transmission light and microscope, a large cooled heat stage could not be used because it would block the light path. Instead, most of the heat needed to dissipate through the side of the chip, into the fixture. Because of this, during the flow sensor measurements, $\mathrm{T}_{\mathrm{c}}$ varies between 17.7 and $23.5^{\circ} \mathrm{C}$ for the subcritical condition, and between 33.0 and $46.8^{\circ} \mathrm{C}$ for supercritical conditions as a result of the heat generated by the high brightness LEDs. For the pressure restrictor measurements, $\mathrm{T}_{\mathrm{c}}$ varied between 15.9 and $16.2^{\circ} \mathrm{C}$. This is not a problem as the internal sensors in the chips directly measure $T_{c}$.

\section{Geometric characterization}

The metallization layers were characterized using scanning electron microscope, SEM, (1550, Zeiss), white light interferometry (Wyko NT1100, Veeco) and optical microscopy (AX70, Olympus). Access to the metal layers exposed to the insides of the channel was achieved by grinding a topside opening to the channels. Cross-sections of the metal layers that were embedded between wafers were done by grinding and etching in $12.3 \mathrm{wt} . \%$ HF for about $10 \mathrm{~s}$. To avoid charging, samples used for SEM were coated with an approximately $6 \mathrm{~nm}$ thick sputtered Pd/Au film.

\section{Measurements}

Fracture tests were done using $\mathrm{CO}_{2}$ that first was pressurized to 80 bar. Capillaries were glued to 
one of the two inlets of each chip and connected to the forward moving pump. The other inlets were plugged with glue. During the pressure tests, the chips were put on cover glass slides without any clamping and no electrical connections were made. The connected capillary was 10 $\mathrm{cm}$ long and $150 \mu \mathrm{m}$ in outer diameter and exerted a minimal mechanical force on the samples. By increasing the pressure at a rate of $0.5 \mathrm{bar} / \mathrm{s}$ until fracture, the fracture pressure was recorded. Chips were held at room temperature during these tests and a high-speed camera was used to follow the fracture behavior. To translate fracture pressure into fracture stress, the channel geometry of the flow sensor was modeled using finite element analysis (Comsol Multiphysics 5.2, Comsol). Leakage measurements were done with water and an external flow sensor (Nanoflow Sensor, IDEX) that was connected to the chips having their outlets plugged. The external flow sensor has a lower detection limit of $1.5 \mathrm{~nL} / \mathrm{min}$. Fluorescent dye has been capable of visualizing nanochannels [34-35]. To see if any leakage could be detected through the possible voids along the metal, samples were filled with an aqueous solution containing $14 \mathrm{~g} / \mathrm{L}$ fluorescein (Sigma-Aldrich), both by means of capillary action and by pressurization to 90 bar. Following depressurization and flushing of excess fluorescein in the channels using water, the chips were observed using a fluorescent microscope (TE2000-U, Nikon).

To calibrate the sensors, the mounted chips and fixtures were put in a convection oven held at $50{ }^{\circ} \mathrm{C}$ (400, Memmert) together with a K-type thermocouple which was used as a temperature reference. The oven was turned off and allowed to cool during $5 \mathrm{~h}$, giving correlated temperatures down to room temperature. The resistance change was linearly fitted against the temperature decrease. The temperature sensor noise was recorded while operating the chips without a pressurized fluid and taken as 2 times the standard deviation.

The pressure drop chip, used to study local temperature variations, was used for two experiments. In the first experiment, the backpressure piston was set to a constant pressure mode and the forward moving piston was set to constant volumetric flow mode. By such action, $\mathrm{P}_{\mathrm{b}}$ was set to 50 or 57 bar and $Q_{a}$ was varied over the range 10 to $100 \mu \mathrm{L} / \mathrm{min}$. The chip temperature, $\mathrm{T}_{\mathrm{c}}$, was measured using the internal temperature sensor located at the chip restrictor inlet. The decompression cooling effect over the restrictor, $\Delta T$, was measured as the absolute difference between the inlet and outlet temperature sensors. In a second experiment, the $T_{c}$ was recorded as a function of time and the forward moving piston was instead operated in constant pressure mode with the backpressure piston held at a fixed volume. Variations in $T_{c}$ were recorded as $P_{f}$ was changed in steps.

For measurements with flow sensor chips, the backpressure piston was again set to hold constant pressure and the forward moving piston was set to constant volumetric flow mode. As a measurement of $T_{c}$, the average temperature of the two sensor elements, denoted $T_{1}$ and $T_{2}$ in figure 3 , was used for calculating a corresponding flow velocity $\mathrm{v}$ for each measurement. As no large $\Delta \mathrm{P}_{\mathrm{fb}}$ is present; $\mathrm{P}_{\mathrm{b}} \approx \mathrm{P}_{\mathrm{c}} \approx \mathrm{P}_{\mathrm{f}}$ and (1) and (3) together with $\mathrm{T}_{\mathrm{c}}, \mathrm{P}_{\mathrm{b}}$ and $\mathrm{T}_{\mathrm{f}}$ can be used to describe $v$. Two measurement series were conducted, with $\mathrm{T}_{\mathrm{c}}$ held either under or over the critical temperature of $31.1^{\circ} \mathrm{C}$. For subcritical conditions, $\mathrm{P}_{\mathrm{b}}$ was set to 80,100 or 120 bar. For supercritical conditions, $\mathrm{P}_{\mathrm{b}}$ was set to either 80 or 100 bar. The heating element was used at 1,3 or $5 \mathrm{~mA}$, producing a power of $0.41,1.23$ and $2.05 \mathrm{~W}$, respectively.

To evaluate the temperature difference, $\Delta \mathrm{T}$, response with $\mathrm{v}$, data was fitted to a nonlinear model of the form,

$\Delta \mathrm{T}=\mathrm{a}\left(1-\mathrm{e}^{-\mathrm{bv}}\right)+\mathrm{c}$,

where a, b and c are constants. To define a statistically relevant working range of the flow sensor 
at a specific condition, the detection limit is determined using the root-mean-square error, $\mathrm{s}_{\text {rmse }}$, of each fitting curve, and it is taken as $2 \mathrm{~s}_{\text {rmse }}$. Given a resolution, $\Delta \mathrm{v}$, in this work set to $1 \mathrm{~mm} / \mathrm{s}$, the upper limit of the working range is calculated from the minimal sensitivity, $\frac{\mathrm{d} \Delta \mathrm{T}}{\mathrm{dv}}$, required to produce a detectable change of $\Delta \mathrm{T}$, i.e. $2 \mathrm{~s}_{\text {rmse}}$.

\section{Results}

\section{Electrode characterization}

The heater and temperature sensors were integrated into the channels of the chip, with sensor elements exposed to the fluid, figure 5 . The electrodes were embedded by letting the metal fill an etched trench in the channel lid, figure 6.

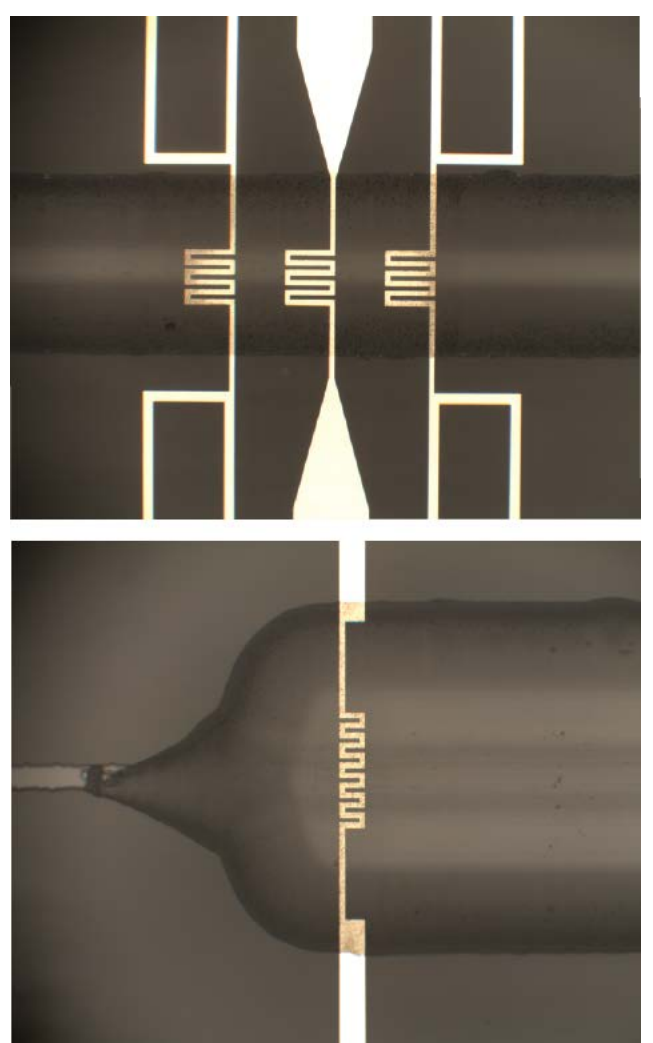

Figure 5 The flow sensor (top) and pressure drop (bottom) device. The smaller sensing area has $10 \mu \mathrm{m}$ wide electrodes.

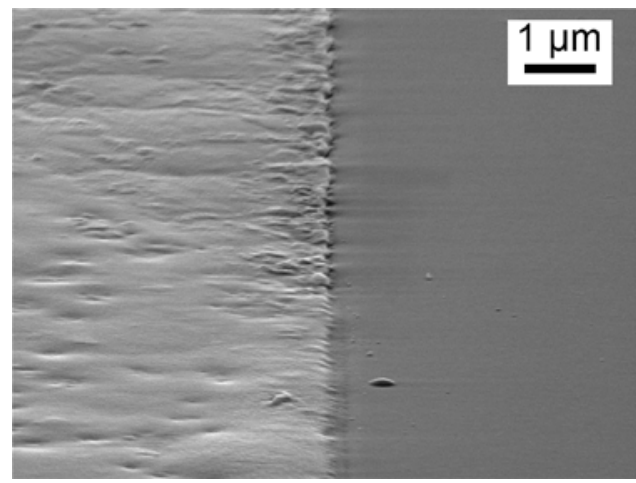


Figure 6 The Pt metal of an electrode in the channel is seen to the left. The glass is seen to the right, with the transition in the middle. The view is tilted $70^{\circ}$ in the direction perpendicular to the metal and glass transition.

Figure 7 shows a height representation of the exposed part of the nominally $10 \mu \mathrm{m}$ wide electrodes in the channels after bonding and thermal treatment. The metal surface had a surface roughness of $4 \mathrm{~nm}$ and larger hills and valleys. Due to these variations, the metal was at most 35 $\mathrm{nm}$ above the glass plane. As this surface topography is inherent to the deposited film, these variations are also present at the bond interface. This implies the presence of gaps with a shape similar to figure $1 \mathrm{~b}$ and a height $\mathrm{d}$ in the range of $35 \mathrm{~nm}$. A trench between the metal and glass was formed, having an average depth of $20 \mathrm{~nm}$ and width of about $2.5 \mu \mathrm{m}$. This trench can also be seen in the tilted view in figure 6 .

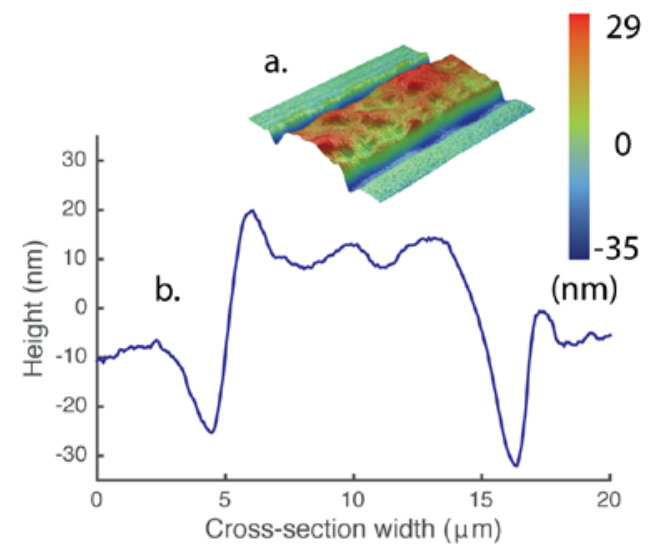

Figure 7 Height representations, (a), of a $10 \mu \mathrm{m}$ wide embedded electrode exposed to the channel inside. The profilometry curve in (b) has valleys on each border of the metallization.

After bonding, the enclosed metallization had no apparent voids or delamination surrounding it when cross-sections of the metal layers were examined using SEM and the glass seems to conform around the metal layer. This is fundamental for leak resistance at high pressure.

When operating the chips with $\mathrm{CO}_{2}$ at 90 bar, no leakage was seen when observing the chips under the stereoscope. Most importantly and conclusive, no fluorescence could be seen either on top or along the metal pathways when the chips were filled with the fluorescent solution. Also, when testing for leakage using the external flow sensor, no flow was registered to keep the pressurization of the chips. This implies that any leakage was below the detection limit of $1.5 \mathrm{~nL} / \mathrm{min}$. The fracture tests showed that fracture occurred at pressures in the range of 80 to 220 bar. The pressure drop and flow sensor chips fractured at pressures between 80 to 173 bar (n $=6)$ and 190 to 220 bar $(n=4)$, respectively. For these measurements, the average fracture pressures were 126 and 201 bar, while the standard deviations were 12 and 35 bar, respectively. Modeling of the flow sensor chip showed that 201 bar corresponds to an average fracture stress of $73 \mathrm{MPa}$. As a comparison, other studies with glass chips with similar geometries but without integrated electrodes showed fracture stresses between 129 and $254 \mathrm{MPa}$ [30]. Both types of chips were operated continuously at 90 bar for more than one week.

By investigation of the fracture surfaces, it was seen that the crack formed during fracture originates from the bond interface edge of the channels. The crack deviates from this interface plane, forming a fracture surface with a topographic height difference from the bond plane of up to $30 \mu \mathrm{m}$. But, in the proximity the channels, the direction of the fracture deviates so that the 
fracture goes along and exposes the metallization layer, showing that the metallization affects the crack growth.

Temperature sensing in the pressure drop chip

Cooling as a consequence of flows through the restrictor on the pressure drop chip was seen, figure 8. The temperature at the inlet was 15.9 and $16.2^{\circ} \mathrm{C}$. With $\mathrm{P}_{\mathrm{b}}$ at $50 \mathrm{bar}$, a $\Delta T$ up to $5.1{ }^{\circ} \mathrm{C}$ was seen as well as a transition from liquid to a gas phase at the outlet, thus forming a multiphase system. With $\mathrm{P}_{\mathrm{b}}$ slightly higher, 57 bar, the gas phase disappeared and a lower $\Delta T$ was observed, around $0.6^{\circ} \mathrm{C}$. This difference was of similar levels also for higher pressures, and depressurization of $\mathrm{P}_{\mathrm{f}}$ from 90 to 70 bar caused a $1 .{ }^{\circ} \mathrm{C}$ drop of $\mathrm{T}_{\mathrm{C}}$ from $13.2^{\circ} \mathrm{C}$ down to $11.4{ }^{\circ} \mathrm{C}$ with a rate of $0.2^{\circ} \mathrm{C} / \mathrm{s}$. The temperature sensor noise was $0.007^{\circ} \mathrm{C}$.

\section{Flow sensing in $\mathrm{CO}_{2}$}

Figure 9 shows $\Delta \mathrm{T}$ as a function of flow rate $\mathrm{v}$ for both sub- and supercritical conditions. Except for the measurement with $2.05 \mathrm{~W}$ at low temperature, subcritical conditions, no measurements at 80 bar could be fitted to a typical flow sensor response explaining the lack of curve fits for most of the 80 bar measurement series. The working ranges of the flow sensor at the specific conditions are presented in table 1 . In the table, the resulting density range for the given condition is presented. Operating the sensor at $2.05 \mathrm{~W}$ yields the largest thermal gradient and the largest range, between 0.5 to $13.8 \mathrm{~mm} / \mathrm{s}$ for a $\Delta \mathrm{v}$ of $1 \mathrm{~mm}$.

While the sensor noise of the flow sensing chip is similar to that of the pressure drop chip, the measurement variation, $2 \mathrm{~s}_{\text {rmse }}$, is much larger, up to $0.057^{\circ} \mathrm{C}$ for the conditions that could be used to fit a curve. This variation is affected by the fluid system and the varying properties of $\mathrm{CO}_{2}$.

At low pressure, boiling occurs at the heater during operation, as exemplified in figure 10 where the pressure is 60 bar and the heating output is $2.05 \mathrm{~W}$. The flow sensor is not working properly when boiling occurs. 


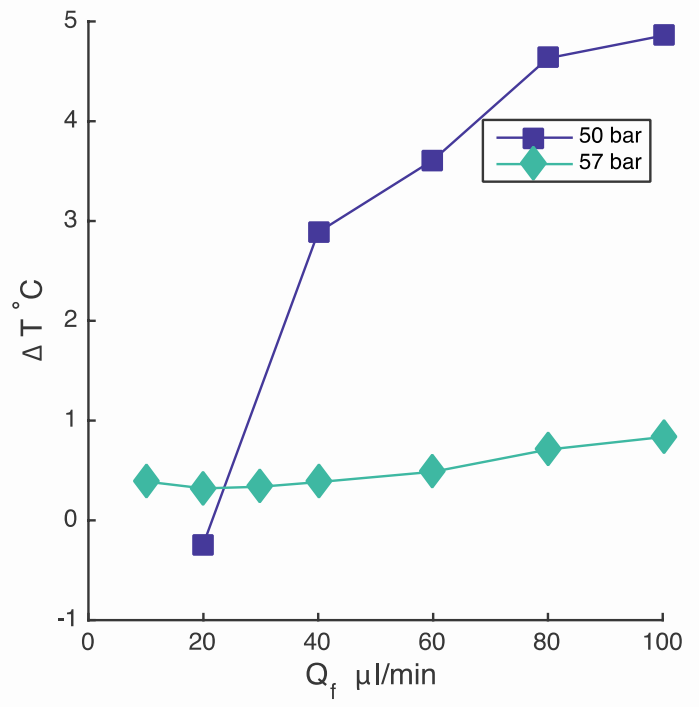

Figure 8 Cooling effect, $\Delta \mathrm{T}$, at the restrictor of the pressure drop chip as a function of $\mathrm{Q}_{\mathrm{f}}$. 

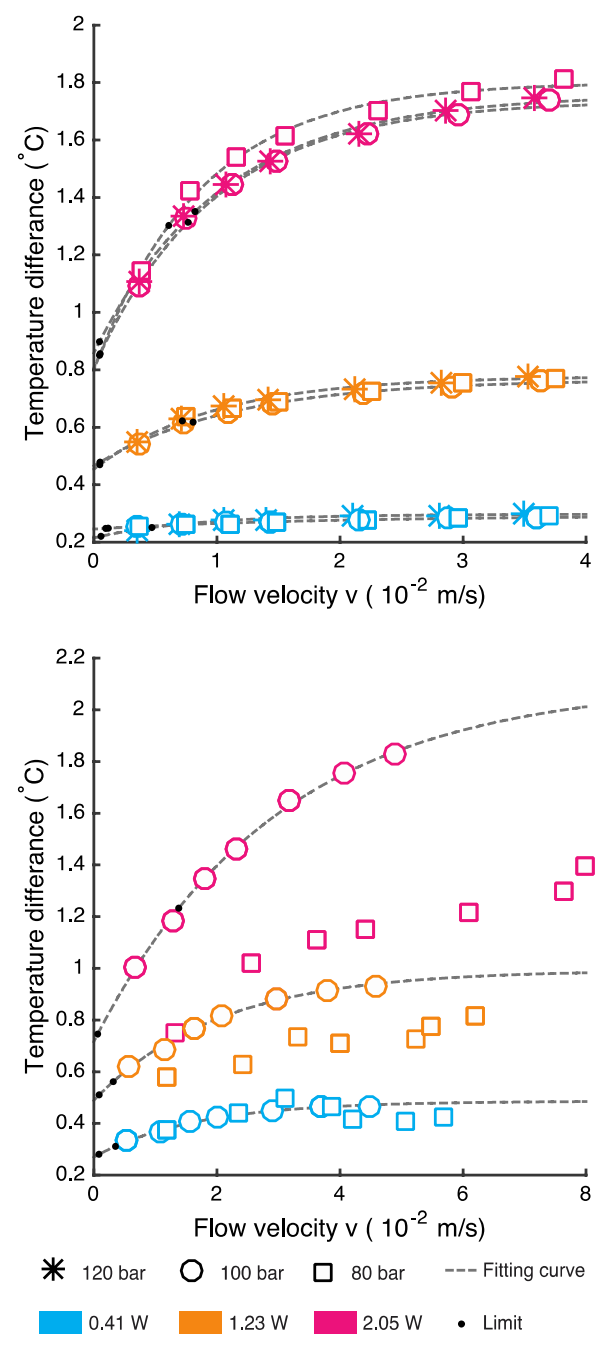

Figure 9 Temperature difference as a function of flow velocity for subcritical (top) and supercritical (bottom) state. Fitting curves with corresponding range limits have been added.

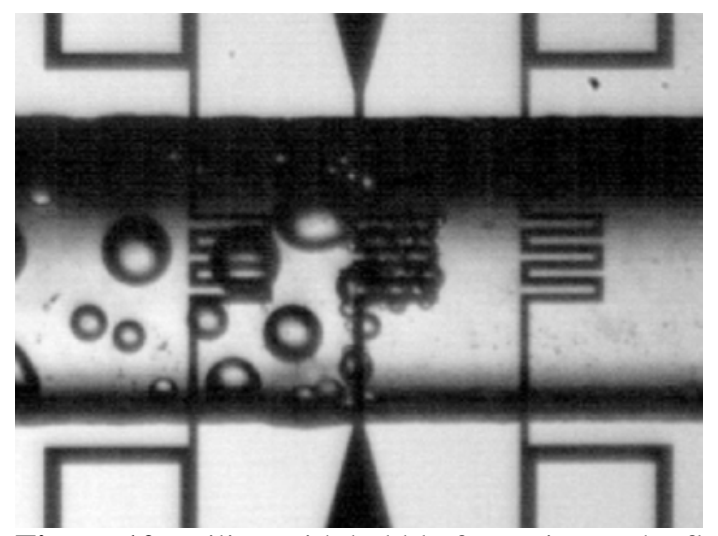

Figure 10 Boiling with bubble formation at the flow sensor heater running at $2.05 \mathrm{~W}$. The pressure is $60 \mathrm{bar}$. The flow goes from right to left in the picture. 
Table 1. Flow sensor range.

\begin{tabular}{ccccc}
\hline & Power $(\mathrm{W})$ & 0.41 & 1.23 & 2.05 \\
\cline { 2 - 5 } $\mathrm{P}_{\mathrm{b}}, \mathrm{T}_{\mathrm{c}}{ }^{\mathrm{a}}\left(\mathrm{bar},{ }^{\circ} \mathrm{C}\right)$ & $\boldsymbol{\rho}_{\boldsymbol{c}}\left(\mathrm{kg} / \mathrm{m}^{3}\right)^{\mathrm{b}}$ & & Range $(\mathrm{mm} / \mathrm{s})$ & \\
\hline $120,17.8-22.3$ & $880-886$ & $0.6-4.7$ & $0.5-7.2$ & $0.5-8.2$ \\
$100,18.2-22.9$ & $854-861$ & $0.1-1.2$ & $0.5-8.1$ & $0.5-7.7$ \\
$80,18.3-22.8$ & $826-834$ & $-{ }^{\mathrm{c}}$ & $-{ }^{\mathrm{c}}$ & $0.5-6.1$ \\
100, 36.3-46.2 & $537-680$ & $0.8-3.5$ & $0.9-3.2$ & $0.5-13.8$ \\
80, 33.5-46.4 & $254-511$ & $-{ }^{\mathrm{c}}$ & $-{ }_{\mathrm{c}}$ & \\
${ }^{\mathrm{a}}$ Maximum and minimum values. & & & \\
${ }^{\mathrm{b}}$ Calculated from $\mathrm{P}_{\mathrm{b}}$ and $\mathrm{T}_{\mathrm{c}}$. & & & \\
${ }^{\mathrm{c}}$ Deviating fitting curve - No range & & &
\end{tabular}

\section{Discussion}

\section{Embedded metal layers}

Insight in the conditions at bonding is given by figure 7. As the etched trench depth and the metallization thickness mismatch, the electrodes are up to $35 \mathrm{~nm}$ higher than the glass, thereby suggesting a situation similar to figure $1 \mathrm{~b}$, where metal is present above the bond layer. While this height is less than the critical size for a spherical particle, the large width of the exposed metal could raise some concerns over the ability to bond. However, no problems have been seen during bonding and the chips can sustain high pressures. The mismatch between the etch trench and the metal deposition results in a $2.5 \mu \mathrm{m}$ wide and $20 \mathrm{~nm}$ deep valleys surrounding the electrodes, but after bonding, this gap was no longer possible to detect in SEM.

While any voids on the nanoscale would allow fluids to flow [35-36], no evidence of leakage has been found either by using fluorescent dye or flow measurements. The lack of any leakage, visible gaps or voids, suggests a mechanism that seals the structure during the thermal treatment. Glass sinters at lower temperatures through viscous flow and a surface energy reduction [37]. $\mathrm{SiO}_{2}-\mathrm{B}_{2} \mathrm{O}_{3}-\mathrm{Al}_{2} \mathrm{O}_{3}$ glass powders have been shown to sinter at their glass transition temperature, $\mathrm{T}_{\mathrm{g}}$, of $624^{\circ} \mathrm{C}$ and have optimal sintering temperatures of $700{ }^{\circ} \mathrm{C}$ [38]. As the $\mathrm{T}_{\mathrm{g}}$ of borofloat glass is $625^{\circ} \mathrm{C}$ [29], both the temperature and time of the heat treatment as well as the dimensions of possible gaps satisfies the conditions for sintering.

Both demonstrator chips withstand the pressures needed for operation around the critical point of $\mathrm{CO}_{2}$. Consistent with the larger geometry of the pressure drop chip, the average fracture pressure was 126 bar compared to the 201 bar achieved for the flow sensor chip. The simpler semi-circular channel geometry of the flow sensor allows for strength-comparisons using modeling to find the correlating fracture stress. The average fracture stress was $73 \mathrm{MPa}$ which is about $40 \%$ less than the stresses seen for chips without electrodes, found in both our previous work [30] and the works of others [39-40]. This and the fact that the crack formation is irregular, following and exposing the metallization, suggest that metallization lowers the fracture strength and the integrated electrodes act as defects. The fracture strength in glass chips is a complex property, not only dependent on the stress, and crack initiating defects are affected by stress corrosion [30]. This makes the device strength also dependent on the time, temperature and environment. While the exact crack formation is unknown, it is prudent to keep both metal structures and channel dimensions small where the electrodes enter the pressurized channels, thus lowering both the 
defect size and local stress.

\section{Measurements of $\mathrm{CO}_{2}$ in a microfluidic system}

The chips clearly demonstrate the possibilities with integrated electrodes for added control of supercritical fluids in microfluidics. Using these systems, direct measurements of the inner wall temperature are made; enabling observations of both the cooling effect at pressure drops and flow velocity measurements up to $0.5-13.8 \mathrm{~mm} / \mathrm{s}$.

It can be confirmed that cooling effects are present while using a restrictor channel on the chip. The boiling point of $\mathrm{CO}_{2}$ is $14.3^{\circ} \mathrm{C}$ at $50 \mathrm{bar}$ and $19.8^{\circ} \mathrm{C}$ at 57 bar. Therefore, the detectable temperature changes of figure 8 at 57 bar represent liquid $\mathrm{CO}_{2}$ expanding as it exits the resistor. Meanwhile, for the measurements at 50 bar, there is also the formation of a gas phase with a connected energy of evaporation. As this forms a non-equilibrated multiphase composition of both gas and liquid $\mathrm{CO}_{2}$, quantitative predictions of the energy output is hard. As multiphase flows are strongly dependent on inertial, viscous and surface tension forces, and with the high variability of such forces for near-critical $\mathrm{CO}_{2}$, getting full control is elusive [17]. Large temperature drops are troublesome for advanced fluid handling where it is common to work close to solubility limits. For several compounds of interest, a drop in temperature of a few degrees can give a supersaturated solution with the compound of interest, and particle formation can result in clogging. Localized temperature sensors as the ones presented here for high-pressure applications give an increased understanding and a precise process control, thereby allowing for optimizations for complex fluid operations.

The flow sensor has been shown to give reliable results at specific conditions. In general, when densities are high, $\Delta \mathrm{T}$ shows lower scattering. However, while operating the sensor at 80 bar and supercritical conditions, the large variation made it impossible to make a normal flow curve fit. This is because a calorimetric sensor response is connected to the heat capacity, $C_{p}$. In figure 11 , it can be seen that at pressures just above the critical point, $\mathrm{C}_{\mathrm{p}}$ shows large variations around a maximum and then decreases with increasing pressure.

For best performance of the flow sensor, sensing should be done either at subcritical conditions or at higher pressures, where both $\mathrm{C}_{\mathrm{p}}$ and density show less variation. As exemplified in this paper, it is, however, possible to extract flow data even at the highly variable conditions around the supercritical point. For an integrated microfluidic platform with several functional units, different temperature or pressure zones on the same chip can be required and conditions around the critical point may present. Due to the highly complex and often non-equilibrated nature of supercritical fluids, the thermal and fluid dynamic aspects of such integrated sensors should be further modeled together with experimental data to give valuable insights. 


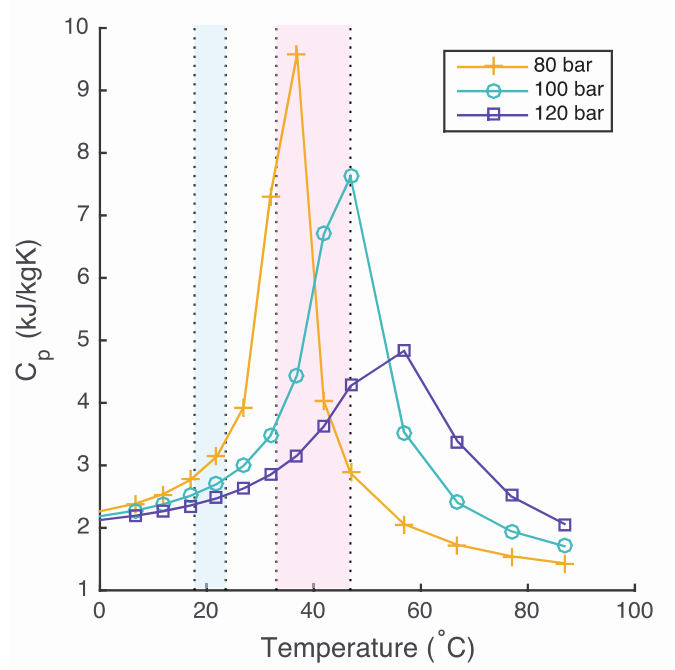

Figure $11 C_{p}$ is shown as a function of temperature [16] for the three different pressures explored in this paper. Temperature ranges $17-23^{\circ} \mathrm{C}$ and $33-47^{\circ} \mathrm{C}$ used for the flow sensor measurements are marked in blue and red zones, respectively.

\section{Conclusion}

Pt electrodes have been integrated into high-pressure glass chips being able to sustain pressures and temperatures for supercritical $\mathrm{CO}_{2}$. Localized cooling effects of up to $5{ }^{\circ} \mathrm{C}$ are seen in a pressure drop chip. Using the integrated, high-pressure tolerant, calorimetric flow sensor, flow sensing under supercritical conditions was done with a workable range up to $0.5-13.8 \mathrm{~mm} / \mathrm{s}$. The integration of thin film sensors adds an essential control to high-pressure microfluidic platforms.

\section{Acknowledgement}

Funding from the Swedish Research Council (contract no. 2011-5037) and the Swedish Agency for the Innovation System, Vinnova, through the Centre for Natural Disaster Science (CNDS), are acknowledged as well as the funding for the laboratory facilities by Knut and Alice Wallenberg Foundation.

\section{References}

[1] Khosravi-Darani K 2010 Research activities on supercritical fluid science in food biotechnology Crit. Rev. Food Sci. Nutr. 50 479-88

[2] Lemasson E, Bertin S and West C 2016 Use and practice of achiral and chiral supercritical fluid chromatography in pharmaceutical analysis and purification J. Sep. Science 39: 21233

[3] Tabernero A, Martín del Valle E M and Galán M A 2012 Supercritical fluids for pharmaceutical particle engineering: Methods, basic fundamentals and modelling, Chem. Eng. Process. Process Intensif. 60 9-25

[4] Tkalec G, Pantić M, Novak Z and Željko K 2015 Supercritical impregnation of drugs and supercritical fluid deposition of metals into aerogels J. Mater. Sci, 50, 1-12

[5] Yeo S D, Kiran E 2005 Formation of polymer particles with supercritical fluids: A review J. Supercrit. Fluids 34 287-308

[6] Dohrn R, Fonseca J M S, Peper S 2012 Exprimental Methods for phase equilibria at high pressures Annu. Rev. Chem Biomol. Eng 3 343-67 
[7] Pinho B, Girardon S, Bazer-Bachi F and Bergeot G 2014 A microfluidic approach for investigating multicomponent system thermodynamics at high pressures and temperatures Lab Chip 143843

[8] Moiseeva E V, Fletcher A A, Harnett C K 2011 Thin-film electrode based droplet detection for microfluidic systems Sensors and actuators B 155 408-14

[9] Moreira N H, de Jesus de Almeida A L, de Oliveira Piazzeta M H, de Jesus DP, Deblire A, Gobbi A L and da Silva J A F 2009 Fabrication of a multichannel PDMS/glass analytical microsystem with integrated electrodes for amperometric detection Lab Chip 9 115-22

[10] Baldwin R P, Roussel T J, Crain M M, Bathlagunda Vijay, Jackson D J, Gullapalli J, Conklin J A, Pai R, Naber J F, Walsh K M and Keynton R S 2002 Fully Integrated OnChip Electrochemical Detection for Capillary Electrophoresis in a Microfabricated Device Anal. Chem 74 3690-7

[11] Eijkel J C T, Stoeri H and Manz A 1999 A Molecular Emission Detector on a Chip Employing a Direct Current Microplasma Anal. Chem 71 2600-6

[12] Sinkala E, McCutcheon J E, Schuck M J, Schmidt E, Roitman M F, Eddington D T 2012 Electrode calibration with a microfluidic flow cell for fast-scan cyclic voltammetry $L a b$ Chip 12 2403-08

[13] Hermes D C, Heuser T, van der Wouden E J, Gardeniers J G E, van der Berg A, 2006 Fabrication of microfluidic networks with integrated electrodes Microsystem. Technogl 12 436-40

[14] Segerink L I, Sprenkels A J, ter Braak P M, Vermes I and van den Berg A 2010 On-chip determination of spermatozoa concentration using electrical impedance measurements $L a b$ Chip 10 1018-24

[15] Sukas S, Schreuder E, de Wagenaar B, Swennenhuis J, van den Berg A, Terstappen L and Le Gac S 2014 A novel side electrode configuration integrated in fused silica microsystems for synchronous optical and electrical spectroscopy Lab Chip 14 1821-3

[16] Span R and Wagner W 1996 A new equation of state for carbon dioxide covering the fluid region from the triple-point temperature to $1100 \mathrm{~K}$ at pressures up to $800 \mathrm{MPa}$ J. Phys. Chem. Ref. Data 25 1509-96

[17] Li S and Tafti D K 2009 Near-critical $\mathrm{CO}_{2}$ liquid-vapor flow in a sub-microchannel. Part II: Flow regimes Int. J. Multiph. Flow 35 1130-7

[18] Kawaji M and Chung P M Y 2004 Adiabatic gas-liquid flow in microchannels Nanosc Microsc Therm 8 239-57

[19] Blanch-Ojea R, Tiggelaar R M, Palleres J, Grau F X and Gradeniers J G E 2012 Flow of $\mathrm{CO}_{2}$-Ethanol and $\mathrm{CO}_{2}$-methanol in a non-adiabatic microfluidic T-junction at high pressures Microfluid Nanofluidics 12 927-40

[20] Assmann N, Werhan H, Ładosz A and Rudolf von Rohr P 2013 Supercritical extraction of lignin oxidation products in a microfluidic device Chem. Eng. Sci. 99 177-83

[21] Knaust S, Andersson M, Rogerman N, Hjort K, Amberg G, L Klintberg and 2015 Influence of flow rate, temperature and pressure on multiphase flows of supercritical carbon dioxide and water using multivariate partial least square regression J. Micromech. Microeng. 25 105001

[22] N.T Nguyen 1997 Micromachined flow sensors-a review Flow Meas Instrum 8 7-16

[23] Wang Y, Chen C, Chang C, Lin C, Lin C, Fu L and Lee C 2009 MEMS-based gas flow sensors Microfluid Nanofluid 6 333-46

[24] Persson A, Lekholm V, Thornell G and Klintberg L 2015 A high-temperature calorimetric flow sensor employing ion conduction in zirconia Appl. Phys. Lett. 106194103

[25] Romaro D F R, Kogan K, Cubukcu A S and Urban G A 2013 Simultaneous flow and thermal conductivity measurement of gases utilizing a calorimetric flow sensor Sens. Actuators, A 203 223-33

[26] Molina A J, Biscarri F, Leal M A, Merino M 2015 Insertion calorimetric flowmeter for 
liquids with multiple temperature sensors to improve measurement by redundancy Flow Meas Instrum 71 58-65

[27] Plößl A and Kräuter G 1999 Wafer direct bonding: tailoring adhesion between brittle materials Mater. Sci. Eng. R-Rep. 25 1-88

[28] Yu H H and Suo Z 1998 A model of wafer bonding by elastic accommodation J. Mech. Phys. Solids 46 829-44

[29] Schott Technical Glass solutions, "BOROFLOAT® 33 - Mechanical Properties ” datasheet, April 2016

[30] Andersson M A, Hjort K, Klintberg L 2016 Fracture strength of glass chips for highpressure microfluidics J. Micromech. Microeng 26095009

[31] Marre S, Adamo A, Basak S, Aymonier C and Jensen K F 2010 Design and packaging of microreactors for high pressure and high temperature applications, Ind. Eng. Chem. Res. 49 11310-20

[32] Heidaryan E, Hatami T, Rahimi M and Moghadasi 2011 Viscosity of pure carbon dioxide at supercritical region: Measurment and correction approach, J. Supercrit. Fluids 56 14451

[33] Williams K R, Gupta K and Wasilik M 2003 Etch rates for micromachining processingPart II J. Micromech. Microeng. 12 761-78

[34] Hoang H T, Tong H D, Segers-Nolten I M, Tas N R, Subramaniam V, Elwenspoek M C 2012 Wafer-scale thin encapsulated two-dimensional nanochannels and its application toward visualization of single molecules J. Colloid Interface Sci 367 455-9

[35] Kuo J and Lin Y 2012 Fabrication of $20 \mathrm{~nm}$ Shallow Nanofluidic Channels Using Coverslip Thin Glass-Glass Fusion Bonding Method Jpn. J. Appl. Phys. 51 095202-5

[36] Cheng J T and Giordano N 2002 Fluid flow through nanometer-scale channels Physical Review E 65031206

[37] Rabinovich E M 1985 Preperation of glass by sintering Journal of Materials Science 20 4259-97

[38] L Dongguo, Yib S, Heob J, Leec J, Germand R M, Park S J 2015 Fabrication of glass components by sintering of commercial glass powder Ceramics International 41 5056-65

[39] Oosterbroek R E, Hermes D C, Kakuta M, Benito-Lopez F, Verboom W, Gardeniers J G E, van den Berg A

2006 Fabrication and mechanical testing of glass chips for high pressure synthetic or analytical chemistry Microsys. Technol. 12 450-4

[40] Tiggelaar R M, Benito-López F, Hermes D C, Rathgen H, Egberink R J M, Mugele F G, Reinhoudt D N, van den Berg A, Verboom Wand Gardeniers H J G E 2007 Fabrication, mechanical testing and application of high-pressure glass microreactor chips Chem. Eng. J. 131 163-70 